\title{
Research on phase transition and strength under ramp compression with graded density impactor
}

\author{
Ye Tan*, Xuemei Li, Yuying Yu, and Ke Jin \\ National Key Laboratory of Shock Waves and Detonation Physics , Institute of Fluid Physics, \\ Chinese Academy of Engineering Physics, Mianyang 621900, China
}

\begin{abstract}
In our work, graded density impactors fabricated from 8 to 40 layers, are specifically designed to generate desired strain rates (on the order of $10^{5} \sim 10^{6} \mathrm{~s}^{-1}$ ) and thermodynamic path(shock loading-ramp loading-release). And experiments on phase transition and strength for metals (bismuth, LY12 Al) have been performed with light gas gun to peak pressure between $30 \mathrm{GPa}$ and $50 \mathrm{GPa}$. Particle velocity at sample/window interface in these experiments are simultaneously traced by a distance interferometer system for any reflector, and a wave profile analysis is employed to explore the solidification transition and strength behaviour along elevated isentrope.
\end{abstract}

\section{Introduction}

Phase transition and strength of materials under extreme conditions is fundamentally interesting in condensed matter physics, Geophysics, some engineer applications and so on $[1,2]$. It is particularly important to describe the characteristics of physical processes and provide the thermodynamic properties of kinetics in material for dynamic compression. Earlier, observations of phase transition and strength in dynamic compression experiments are frequently made via satisfactory techniques, gas gun, explosive, powerful pulse facilities and so on. Despite kinds of experimental and theoretic methods have been applied to obtain the basic properties of materials, however, it seems that the existing experimental and theoretical describing characteristics for materials at a wide pressure-temperature-strain rate range are still surprisingly puzzled and give a great variety for model verification, such as the large unexplored region of phase space among Hugoniot, isentrope and static experiments. In order to define the additional information of materials beyond the principal Hugoniot and isentrope, a controlled-path loading method based on graded density impactor (GDI) are employed here. And shock-ramp loading -ramp release experiments were performed on bismuth and LY12 Al to detect the phase transition and strength behavior.

\section{Experiment}

In this work, the controlled path generated with graded density impactor was designed for the dynamic behavior of materials, including solidification of bismuth and strength of LY12 $\mathrm{Al}$ at different strain rate. And the density profiles that can provide increasing shock impedance was designed as the equation $\rho=\rho_{0}+A x^{p}$, which have been proven to be more suitable to generate ramp pressure pulses by Aditi Ray [3]. Aluminum, magnesium, titanium, copper, tungsten were chosen as the standard materials for graded density impactor, and every layer of the impactor can be made of any materials or any combinations thereof to extend the density from aluminum to tungsten continuously ( As shown in Fig.1). The impactor here we used typically have between 8 to 40 layers of $200 \mu \mathrm{m} \sim 1000 \mu \mathrm{m}$ thick at the diameter from $20 \mathrm{~mm}$ to $50 \mathrm{~mm}$.

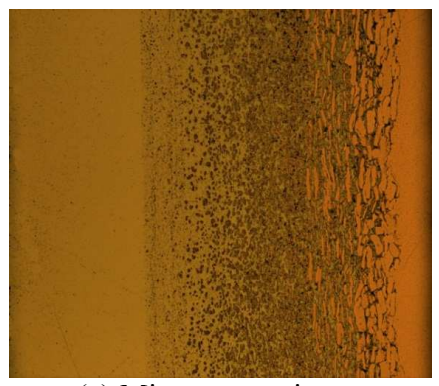

(a) Microscopy picture

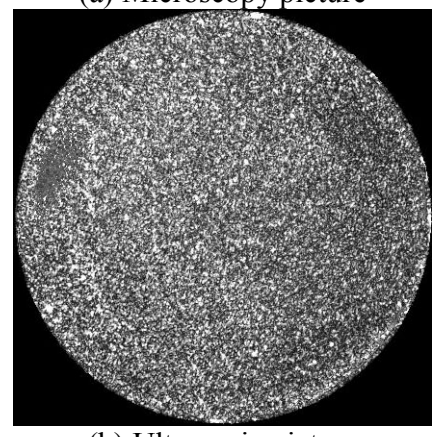

(b) Ultrasonic picture

Fig. 1. Scanning microscopy and ultrasonic picture of $\mathrm{Al}-\mathrm{Cu}$ impactor before the experiment. 


\subsection{Experiment of bismuth}

To cause bismuth melting with the initial shock loading generated by the first layer of graded density impactor and then solidification with the subsequent ramp loading generated by the other layers of graded density impactor, impedance for every layer was designed with MLEP (Multi-Material Lagrangian Elastic-Plastic) code and the complete equation of state built by Johnson ${ }^{[4]}$. And the corresponding pressureparticle velocity-time profile and thermodynamics loading path for a 17-layer graded density impactor made of Ti-Cu-W was shown in Fig. 2 and Fig. 3.

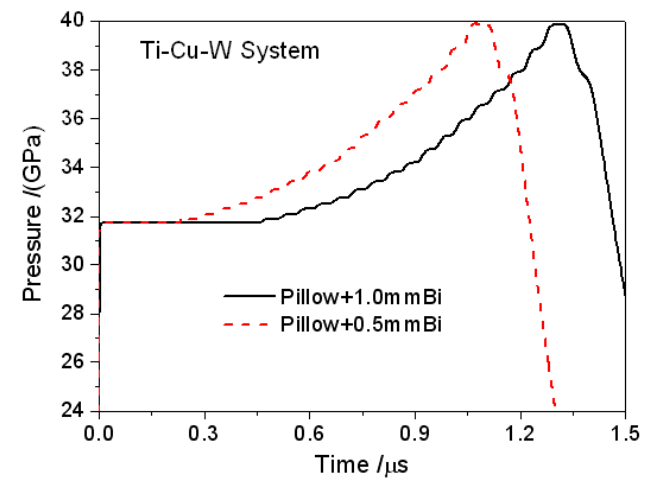

(a) Pressure history

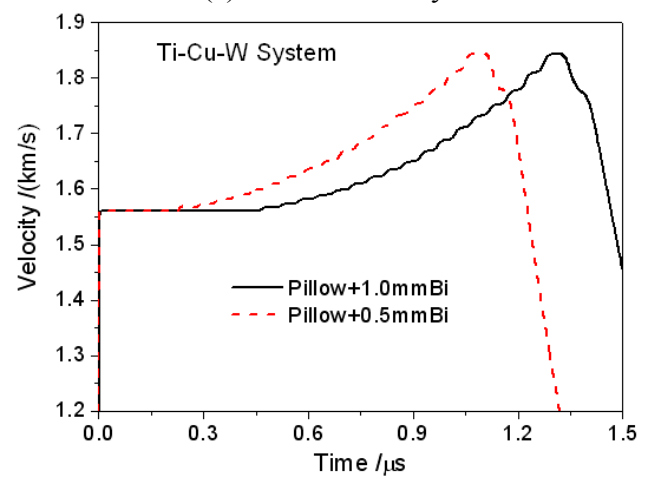

(b) Particle velocity history

Fig. 2. The calculated pressure and particle velocity history for solidification of bismuth.

The idealized experimental configuration of typical controlled path experiment for bismuth was presented in Fig. 4. The graded density impactor glued with a $1 \mathrm{~mm}$ bismuth was accelerated to impact a $10 \mathrm{~mm} \mathrm{LiF}$ window, with a $30 \mathrm{~mm}$ two stage light gas gun. These windows are coated with a thin metallic foil $(\sim 8 \mathrm{um} \mathrm{Al})$ and the particle velocity profile was traced with a distance interferometer system for any reflector (DISAR) at the impact interface. The measured particle velocity with the simultaneous result is shown in Fig. 5. As shown in the figure, the initial impact loading was applied on bismuth with the first layer causing melting, then the particle velocity decrease after about 0.55 us (may separate between graded density impactor and bismuth), and as the reflect wave generated by other layers of graded density impactor caught up with shock wave in bismuth, the particle velocity increased smoothly, suggesting the solidification by the ramp loading, which have been predicted by the simulation shown in Fig. 5 and Fig. 6 .

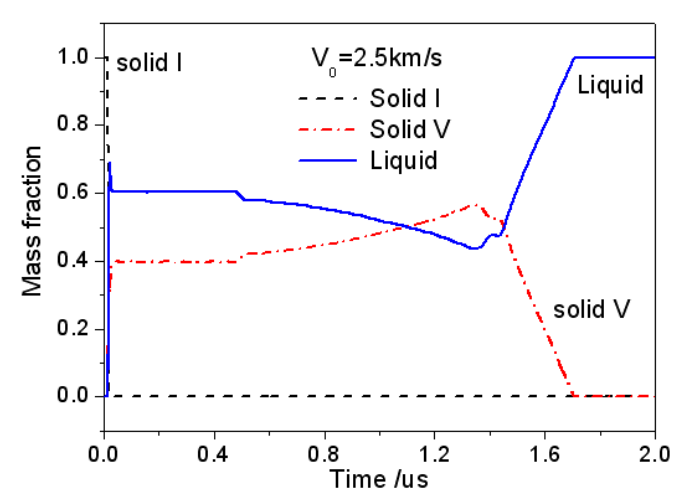

(a) Mass fraction history

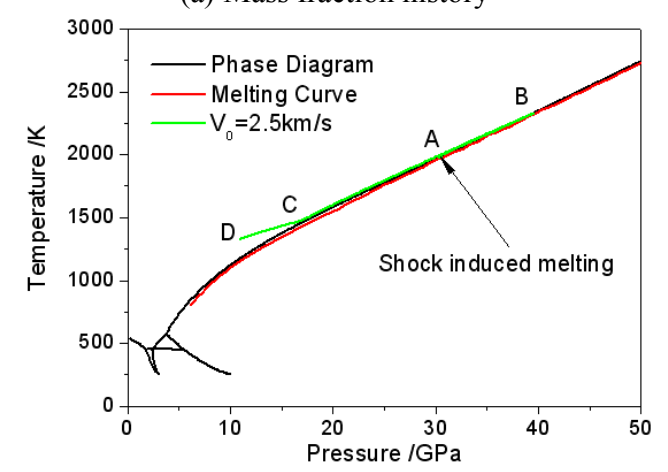

(b) Temperature history

Fig. 3. The calculated thermodynamic path for solidification of bismuth.
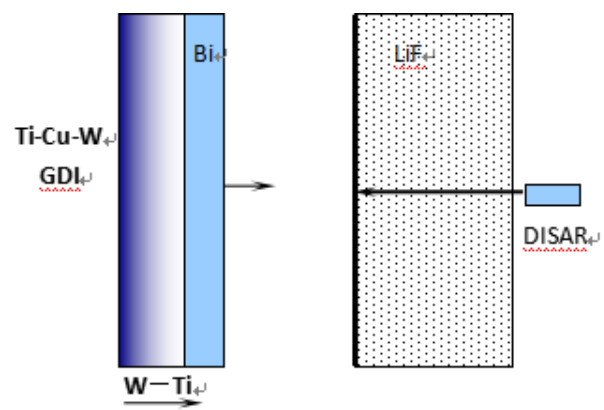

Fig. 4. The idealized experimental configuration for solidification of bismuth.

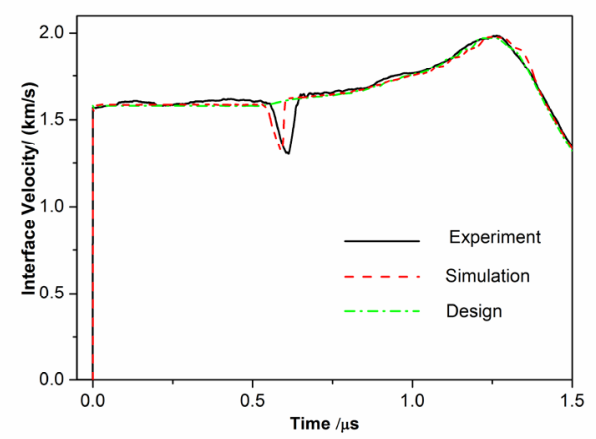

Fig. 5. Comparison of velocity profiles among experiment, simulation and design results. 


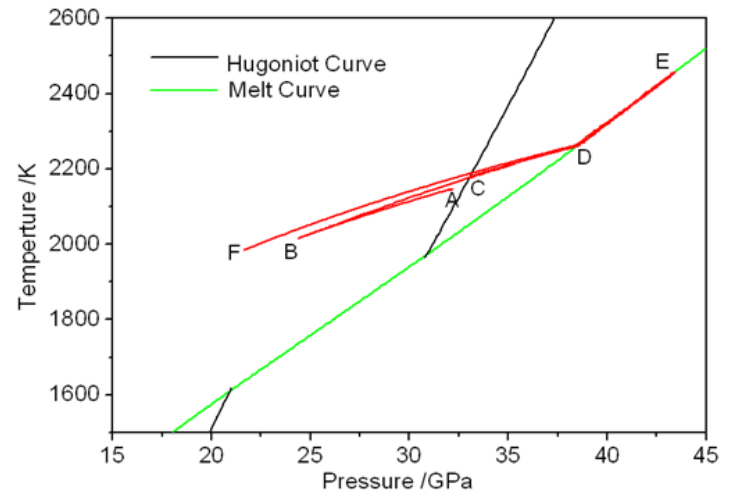

Fig. 6. Thermodynamic path for solidification of bismuth.

\subsection{Experiment of LY12 Al}

Based on the ability to control the loading path, the graded density impactor were also employed to control the pressure, temperature, strain rate and explore strength behavior of materials under uniaxial strain dynamic loading. Here, a 12-layer graded density impactor made of Al-Cu was used to generate $\sim 5 \times 10^{5} \mathrm{~s}^{-1}$ strain rate and obtain yield strength data of LY12 Al with self-consistent method (Fig. 7). The graded density impactor was accelerated to impact stepped LY12 Al glued with a $10 \mathrm{~mm} \mathrm{LiF}$ window on a $30 \mathrm{~mm}$ two stage light gas gun. The particle velocity profile was traced with DISAR at the interface.
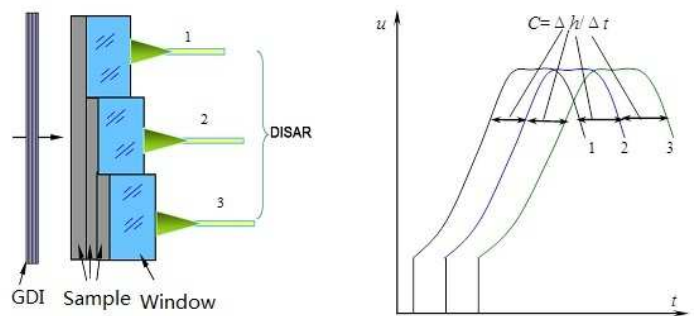

Fig. 7. The idealized experimental configuration for strength experiment of LY12 Al.

The measured particle velocities are shown in Fig. 8. As the launched flyer impacted the samples, shock wave generated by the first layer passed through the sample into the sample/window interface, conducting discontinuities for the particle velocity and staying with a flat for $\sim 20 \mathrm{~ns}$, then the reflect wave generated by other layers travelled across the sample with designed strain rate, causing the ramp loading, a 10-ns flat and the subsequent release state. On the other hand, the simulated results with MLEP code, which were satisfied with our experiment results, were also presented in Fig. 8. Then, a transfer function methodology was used to account for the complex wave interaction and get the in situ velocities [5]. Graded density impactor performed on LY12 ramp compressed to peak stress of $47.3 \mathrm{GPa}$, with yield strength data at $\sim 1.3 \mathrm{GPa}$.

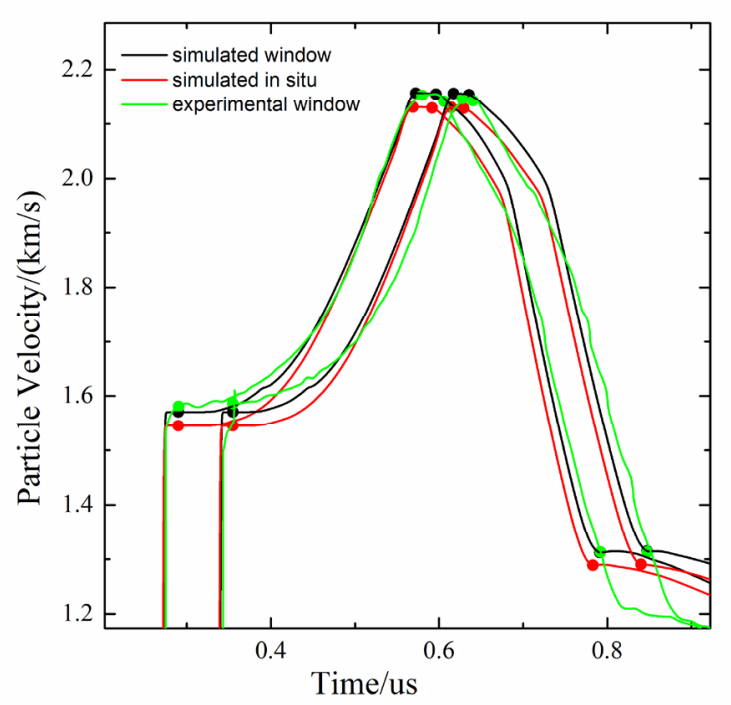

Fig. 8. Comparison of velocity profiles among experiment, simulation results.

\section{Conclusions}

The graded density impactors were used for dynamic behavior of materials under uniaxial strain dynamic loading up to high pressure and high strain rate. Improvement are nessary and possible in the future with new technology and other experimental configuration.

\section{References}

1. G. E. Duvall and R. A. Graham. Rev. Mod. Phys. 3,523 (1977)

2. B. A. Remington, et al. UCRL-JRNL-215701, (2005)

3. A. Ray and S. V. G. Menon. J. Appl. Phys. 110, 024905 (2011)

4. J. N. Johnson, et al. J. Appl. Phys. 46, 3438 (1975)

5. J. L. Brown, et al. J. Appl. Phys. 114, 223518 (2013) 
